\title{
EDIFICACIÓN Y SISTEMA COMO PENSAMIENTO. LA LECTURA HERMENÉUTICA DE LOS TEXTOS FILOSÓFICOS
}

\author{
Simón RoYo HERNANDEZ \\ $U N E D$
}

\begin{abstract}
RESUMEN. Dos modelos de interpretación jalonan la historia de la recepción moderna de los textos filosóficos, una forma de lectura edificante y otra sistemática. Mientras que la primera habita en la pluralidad de las interpretaciones y maneja diversas formas de verdad, la segunda sigue anclada en la búsqueda de una verdad única que habría de brotar homogénea desde el fondo de la página escrita. Los dos modelos conforman ya una potente tradición contrapuesta y, aunque la edificación no vaya después del sistema sino que sea un procedimiento distinto, es necesario subrayar que la filosofía edificante ha crecido haciéndose cargo de la filosofía sistemática; no rechazándola, ni continuándola, sino repensándola y situándola así en el lugar que le corresponde, como un caso particular dentro de una pluralidad de formas del pensar. Un momento dogmático que no resulta descartable ni peligroso mientras no se implante como hegemónico.
\end{abstract}

Hay dos presupuestos filosóficos que influyen a la hora de plantearse y abordar una lectura, sea ésta filosófica o de cualquier otra disciplina cognoscitiva. El primero está relacionado con la filosofía tradicional y el segundo con la filosofía postmoderna. Esos dos presupuestos son los siguientes:

1. Existe un terreno de lo teórico, el pensamiento puede alcanzar verdades y realizar juicios objetivos, separándose del nebuloso ideológico que circunda las mentes humanas; es decir, se puede llegar a separar la ciencia de la ideología, ya sea total o parcialmente, en mayor o menor grado. Se pueden separar los resultados (ciencia, contexto de justificación) del desarrollo que ha llevado hasta ellos (historia, contexto de descubrimiento), al menos a efectos cognoscitivos, que son los que aquí interesan. 
Dentro del presupuesto de la verdad objetiva (lo que desde el plano de la Ontología se ha venido a llamar Metafísica) hay tres variantes principales:

A) El conocimiento se realiza cuando se llega hasta el terreno de los conceptos donde se alcanzan las verdades en la forma de una teoría. La Lógica es la esencia del conocimiento y la base de todo conocimiento en general, pues aunque la mente sólo se llena con experiencias, se llena ordenadamente, de acuerdo con la estructura lógica con la que está provista de antemano. Las teorías tienden a articularse en un Sistema acumulativo de pretensiones omnicomprensivas pero siempre abierto a la incorporación de nuevas teorías y a la corrección de las antecedentes. La tradición que avala este punto de vista es lo que generalmente se denomina como platonismo y defiende un cierto espacio trascendental.

B) El conocimiento se realiza cuando se captan hechos brutos indubitables o cuando se acumulan suficientes hechos a favor de una propuesta, o cuando se cuenta con los suficientes hechos del presente con los que elaborar una propuesta sobre la que no quepa duda razonable; hasta que sean desmentidos los antiguos por otros nuevos y haya que rehacer la teoría. La esencia del conocimiento es entonces la experiencia y la mente es una tabula rasa cuyo primer ingrediente es ese espacio vacío dispuesto para llenarse. La tradición que sustenta este punto de vista procede del inductivismo y del positivismo lógico, desde donde se destaca un único espacio de inmanencia para el ser y el pensar.

C) La fusión de los dos anteriores. Teoría y experiencia son igualmente esenciales para el proceso de conocimiento y es sólo a través de ambas como se alcanza la verdad.

a) La primera forma del pensar es racionalista y teoreticista y defiende un espacio trascendental, previo, separado o separable del de las experiencias; b) la segunda forma del pensar es inductivista y defiende que no hay espacio previo, siendo por agregación de experiencias que se construye el espacio separado o separable, que será posterior; $\mathrm{y}$ c) la tercera resulta una combinación de ambas, basada en la distinción entre lo que es primero gnoseológicamente (Lógica y Teoría de la Ciencia) y lo que es primero existencialmente (Experiencia y hechos del presente), donde se puede defender que pese a ser ambos momentos igualmente necesarios para el proceso de conocimiento, la captación de lo verdadero es algo relacionado, principalmente, con la parte gnoseológica o teorética más que con la 
parte sensible o perceptual. Dicho de otro modo, a los efectos de la epistemologia se nos demostraría que la parte más importante de la teoría de la verdad sería la Lógica, como habrían indicado unilateralmente los teoreticistas, aunque a los efectos de la vida existencial lo fundamental serían las experiencias.

No puede decirse respecto de la propia vida existencial de la teoria que ésta no sea un hecho entre los hechos o, más bien, una experiencia compleja particular, no subsistente (existencialmente) con independencia de la vida, pero sí puede decirse a los efectos del propio carácter gnoseológico de la teoría, que pueden y deben plantearse las cuestiones (teóricamente) con independencia de su pertenencia inmanente al mundo de la vida. Punto éste relacionado con la cuestión del margen o espacio de la justificación frente al contexto de descubrimiento, a fin de no reducir el primero al segundo, la estructura a su génesis, el presente a su historia, reduccionismo que nos llevaría, de lleno, al punto siguiente.

2) No hay espacio al margen de la historicidad ni, por tanto, de lo ideológico. No se puede distinguir entre ciencia e ideología porque las ciencias son siempre ideológicas y las mentes se construyen lingüística y contextualmente en un espacio pragmático concreto. No puede hablarse entonces de verdad ni de juicio objetivo sino tan sólo de acuerdo convencional entre partes ideológicas alcanzado por la vía de la argumentación en una situación ya dada, de acuerdo con los resultados pragmáticos o ventajas (intereses) que quieren y obtienen los dialogantes o lectores de la realidad o de un texto cualquiera. Sólo hay interpretaciones, lecturas cargadas de ideología o historia contextual, que en ese sentido son relativas e inconmensurables unas respecto a las otras, aunque puedan cotejarse, compararse y pueda elaborarse un criterio (a su vez hermenéutico y temporalmente caduco) de mejor o más competente interpretación: «Un mismo texto permite incontables interpretaciones: no hay una interpretación "correcta" ${ }^{1}$. Un escrito es ya una interpretación y a partir de una interpretación sólo se puede sacar otra. Y aunque en Gadamer quede abierta la puerta para la búsqueda trascendentalista de constantes de la interpretación, toda lectura estará determinada por una pluralidad de coordenadas materiales variables:

\footnotetext{
${ }^{1}$ Friedrich NIETZSCHE, Nachgelassenne Fragmente 1885-1886, 1 [120].
} 
La interpretación tiene que dar con el lenguaje correcto si es que quiere hacer hablar realmente al texto. Por eso no puede haber una interpretación correcta "en sí», porque en cada caso se trata del texto mismo. La vida histórica de la tradición consiste en su referencia a apropiaciones e interpretaciones siempre nuevas. Una interpretación correcta en sí sería un ideal desideado, incapaz de conocer la esencia de la tradición. Toda interpretación está obligada a someterse a la situación hermenéutica a la que pertenece ${ }^{2}$.

Luego, si hemos de vivir con interpretaciones, por lo menos procuremos alcanzar a) la más bella (edificación) o b) la más ventajosa (pragmatismo) y la más justa (moralismo), ya que la idea de alcanzar la $\mathrm{c}$ ) verdadera (ciencia) era una fantasía, una ilusión, cuando no una ideología perniciosa y dogmatizante. Galileo dijo desde el punto de vista moderno que el texto de la Naturaleza estaba escrito en lenguaje matemático y hoy vemos que numerosos lenguajes además de los científicos (naturales, estéticos, musicales) reclaman también su capacidad de leer en el libro del mundo con acierto. Para el punto de vista postmoderno las verdades son acuerdos, construcciones histórico-culturales conscientes, cuando no subterráneas construcciones histórico-culturales inconscientes de las que llamamos verdad a su acontecer ante la conciencia.

1.1) Ahora bien, fácilmente puede apreciarse que cuando leemos un texto a través del cual queremos adquirir conocimientos, si es que hay una verdad que desentrañar por medio de la lectura, ésta tendrá que tender a ser univoca, esto es, que cualquiera que lea bien tendrá que lograr desentrañar más o menos la misma información y adquirir más o menos los mismos conocimientos que otro. La idea de la posibilidad de lectura objetiva pertenece al presupuesto de la filosofía tradicional y al presupuesto de que hay verdad. Sin embargo, la pretensión de lectura objetiva de la filosofía se divide al menos en tantas áreas como quedaron precisadas anteriormente: a) la lectura teoreticista, que sería la más propiamente filosófica y que se caracterizaría por procurar no mezclar las lecturas filosóficas con las científicas ni ideológicas, donde se entenderá por lecturas filosóficas las que traten de lo más general o universal (onto-logía o lógica del ser) para diferenciarlas de las lecturas de las disciplinas particulares; b) la lectura inductivista, 477.

${ }^{2}$ Hans-Georg GaDAMER, Verdad y método I, Ediciones Sígueme, 4. ed., Salamanca, 1991, p. 
que consistirá en progresar desde las disciplinas particulares, las ciencias o la literatura, hacia la comprensión de los textos más específicamente de filosofía, eminentemente a partir de las disciplinas históricas y filológicas por parte de los académicos actuales; y c) la lectura teorético-inductiva, la que combinará el análisis histórico-filológico o el uso de cualquier otra disciplina particular (científica, histórica o literaria) con ese ir a las cosas mismas o lectura directa y pura anterior, a la hora de leer y procurar comprender un texto filosófico. El presupuesto de la conmensurabilidad o intersubjetividad de las mentes es aquí tan esencial como el de su inserción en el mundo.

Mediante las determinaciones históricas se buscarán las necesidades externas del texto y mediante la lectura directa las necesidades internas (o lógicas) del mismo. Obviamente desde el paradigma de la verdad y tratándose de la lectura objetiva de la filosofía lo segundo será lo más importante y esencial, mientras que lo primero será secundario ( $\mathrm{y}$ viceversa, como veremos a continuación). La finalidad de estas lecturas será alcanzar la verdad del texto, esto es, alcanzar la captación de la verdad objetiva (ya sea que se alcance en cuanto objetivación o se nos aparezca en cuanto acontecimiento) y ya no tanto la producción de nueva verdad, como ocurre en la lectura edificante.

2.1) No hay verdad objetiva y no hay hechos, sino sólo interpretaciones, que no son propiamente ni lo uno ni lo otro. Al leer un texto filosófico o cualesquiera otro lo que tenemos que lograr con ello es: a) nuestra edificación, esto es, nuestra educación moral o estética, el cultivo de nuestra sensibilidad o b) el pragmatismo, la utilización de las interpretaciones que hagamos de ese texto para fines socialmente relevantes, además del cultivo de la razón pragmática estableciéndose convenciones que se tendrán por verdaderas y funcionarán como tales, pero que no habrán de serlo en realidad (por no haber ninguna realidad última inmutable a alcanzar ni, por consiguiente, ningún acceso a la misma). Para la lectura filosófica se podrán utilizar las disciplinas filológicas e históricas, pero no porque haya una única verdad histórica o filológica que nos ayude a desentrañar la verdad del texto, sino al contrario, o bien para dejar constancia de la no-verdad del texto (deconstrucción), desvelamiento de lo no-dicho pero requerido por un texto; o bien para nuestro estímulo como material para la construcción, en conversación con los demás, de las verdades pragmáticas (útiles y necesarias a la sociedad) o estéticas (hermosas, morales y educativas) del presente, que serán relativas al contexto de su entorno y momento de su construcción. La finalidad de la lec- 
tura hermenéutica de la filosofía no es la captación de la verdad del texto, como medio de captar la verdad objetiva, sino la obtención de sugerencias para la construcción social del presente, para el alumbramiento de otras verdades posibles, para la constitución de nuevos modos de ser.

3) Para ilustrar los dilemas de la lectura hermenéutica de los textos filosóficos vamos a acudir a un caso particular, las lecturas de Nietzsche, y, en concreto, a su lectura por Heidegger, autor este último al que se tiene por uno de los padres de la hermenéutica, siendo él quien estudia a otro de los tenidos como tal.

Quisiéramos resaltar primero, acerca de la lectura que Heidegger hace de Nietzsche, cómo se nos dice que lo que se busca es una confrontación que facilite la tarea del pensar:

Confrontación es auténtica crítica. Es el modo más elevado y la única manera de apreciar verdaderamente a un pensador, pues asume la tarea de continuar pensando su pensamiento y de seguir su fuerza productiva [wirkende - efectiva] y no sus debilidades. ¿Y para qué esto? Para que nosotros mismos, por medio de la confrontación, nos volvamos libres para el esfuerzo supremo del pensar.

Heidegger, por tanto, no expone a Nietzsche para que lo conozcamos, sino que intenta desarrollar lo productivo (efectivo) de su pensamiento, restar sus debilidades y aprovechar sus reflexiones para realizar su propia labor de pensamiento. Pero entonces ¿por qué hablar de Nietzsche? Si se lee de forma edificante, para estimular el propio pensamiento y no para captar y exponer la verdad de los pensamientos ajenos, ¿para qué mencionar a ninguno de los leídos? Y ¿por qué no mantener el anonimato? Cuando alguien se ha apropiado de los pensamientos de otros ni siquiera es consciente, al escribir, de que sus reflexiones proceden de las fuentes por las que transitó. La lectura edificante se diferencia de la lectura filosófico-tradicional o de la lectura erudita (corolario y determinación de la anterior) como el género del Ensayo del de la Tesis doctoral. ¿Acaso como

${ }^{3}$ MarTin HeIdegGer, Nietzsche I, "Nietzsche como pensador metafisico», Editorial Destino, Barcelona, 2000, p. 21. 
argumento de autoridad? ¿Es que no nació la propia hermenéutica en el seno de las exégesis de los textos sagrados?

Desde la lectura heideggeriana de Nietzsche, entonces, vemos que un pensador al leer a otro busca su fuente más pregnante para beber de ella y alimentarse de su savia. Heidegger piensa haber encontrado la de Nietzsche en sus fragmentos más controvertidos. Y se podrá discutir la postura heideggeriana en cuanto afirma que lo esencial del pensamiento de Nietzsche se encontraría en su proyectada obra, nunca llevada a efecto (excepto por la compilación de su hermana nazi), La voluntad de poder. Parecería como si Heidegger aceptase en este punto que el filósofo cuando da lo mejor de sí mismo es al final, después de muchos años de reflexión, pero eso le da a la tarea del pensar un progresismo un tanto hegeliano, un progresismo del que, en realidad, es difícil escapar. Sin embargo, en otras obras, Heidegger ha rechazado esa visión acumulativa o de progreso; me refiero a los Holzwege, cuando en La época de la imagen del mundo nos dice Heidegger que nadie diría que las tragedias de Shakespeare fuesen un progreso respecto a las de Esquilo. Al cuestionar la idea de progreso de la historia ¿no habría de poner en suspenso también la tan manida idea de progreso intelectual biográfico? Si las lecturas son inconmensurables no se puede progresar por medio de las reflexiones de otros sino que habrá que pensarlo todo, de nuevo, uno mismo. Sin embargo es precisamente Hegel quien plantea que hay que hacer las dos cosas, o, mejor dicho, que las dos cosas son lo mismo: hay que pensarlo todo uno mismo, transitar por todas las figuras de la conciencia, pero así no se hace sino aprovechar el acumulado caudal cultural de la humanidad.

El que Nietzsche pertenezca a la tradición en cuanto metafísico, en cuanto propugnador de una metafísica de la voluntad de poder, sería, a juicio de Heidegger, su debilidad; mientras que en sus tesis sobre el Eterno Retorno estaría su fuerza productiva. Ése es el resultado final de la propia confrontación de Heidegger con Nietzsche. ¿Qué ocurre si nuestra propia confrontación con el pensamiento de Nietzsche no nos lleva a esa misma conclusión? ¿Hemos de seguir la lectura de Heidegger? ¿Tendremos que confrontar nuestro pensar a Nietzsche con el pensar a Nietzsche de Heidegger? ¿O acaso serán las lecturas inconmensurables? ¿Cómo contrastarlas, cómo evaluar qué lectura comprende y cuál no comprende? ¿Cuál de las versiones e interpretaciones será competente y cuál no? ¿Cuál lo será más y cuál menos? 
No sabemos, por tanto, si lo que debe hacerse con el Nietzsche de Heidegger es lo mismo que Heidegger hizo con el propio Nietzsche. La actitud puede ser de dos maneras: a) Leer como eruditos, esto es, como estudiosos de Nietzsche, lo que nos obligará a revelar que Heidegger no le hace justicia a Nietzsche, no es (quizá nadie puede serlo si aceptamos ciertos principios de la hermenéutica) un estudioso que pretende exponer neutralmente a los legos y expertos el pensamiento de Nietzsche, esto es, la verdad del texto nietzscheano, como pretenden hacer Giorgio Colli ${ }^{4}$ o Walter Kaufmann ${ }^{5}$, por citar sólo a dos de los mejores estudiosos de la obra nietzscheana. b) Podemos leer como filósofos, recoger la fuerza productiva del pensamiento de Heidegger sobre Nietzsche y descartar sus debilidades. Pero podemos hacer esto último tanto de forma edificante como de forma sistemática.

En ese último caso nos parece que la calificación de Nietzsche como pensador metafísico es una de las debilidades de la lectura de Heidegger, es más, llegaríamos hasta a afirmar que la idea de olvido del ser desde los presocráticos hasta el propio Heidegger se nos presenta como una debilidad del pensamiento de Heidegger ( $y$ no su fuerza productiva). Y para ello podemos volver a citar al propio Heidegger, cuando dice que "todos los grandes pensadores piensan lo mismo. Pero este mismo es tan esencial y tan rico que ninguno puede agotarlo», ${ }^{6}$ añadiríamos, ni siquiera Heidegger, lo que quizás esté implícito en su filosofía, pero no en quienes la siguen. El hecho de haber puesto el ser en un determinado ente en particular, la ontificación del pensamiento efectuada por numerosos pensadores, no resta a su reflexión alcance ontológico, ya que desde los presocráticos seguimos sin estar seguros de si el ser no estará esencialmente relacionado con determinado ente u entes. $Y$ el problema que Heidegger soluciona mediante la diferencia ontológica no responde a la pregunta de si el ser tiene relación con un ente $u$ otro, responde que la relación no es determinante sino que el ser es la posibilidad de todos los entes, cosa que han sabido todos los grandes filósofos. Pero sin determinaciones la filosofia no se arriesga y se convierte en irrefutable, tan irrefutable como formal, tan incontrovertible como abstracta, tan idealista como pura.

${ }^{4}$ Giorgio Colli, Scritti su Nietzsche, Adelphi Edizioni, Milán, 1993.

5 Walter Kaufmann, Nietzsche. Philosopher, Psychologist, Antichrist, Princeton, Princeton University Press, 1974.

${ }^{6}$ HeIdegGer, op. cit., p. 46: "El ser del ente como voluntad en la metafísica tradicional». 
Heidegger menciona de forma despectiva las lecturas de Nietzsche de un erudito (A. Baeumler) y un filosofo (K. Jaspers), principalmente porque ambos, insiste, presentan dos interpretaciones que no han comprendido la doctrina del eterno retorno. El primero, porque ha pretendido una interpretación política de Nietzsche; "la doctrina nietzscheana del eterno retorno choca entonces con la concepción baeumleriana de la política. Por tanto, esa doctrina carece de importancia para el sistema de Nietzsche. Esta interpretación resulta tanto más sorprendente por cuanto Baeumler es uno de los pocos que han atacado la comprensión psicológico-biologista de Nietzsche desarrollada por Klages» ${ }^{7}$. El segundo, según Heidegger, no ha comprendido pues «no es posible tomar en serio la doctrina como una cuestión de contenido porque para él no hay en la filosofía una verdad del concepto y del saber conceptual ${ }^{8}$. Es decir, que a Baeumler le pierden sus prejuicios politicos y a Jaspers sus prejuicios filosóficos a la hora de leer a Nietzsche. Y al final son todos los demás quienes no comprenden, porque los demás filósofos, según Heidegger, no llevan «este pensamiento al ámbito de la pregunta fundamental de la filosofía occidental» ${ }^{9}$, esto es, no consideran el ser como apertura ni consideran la verdad como el estado de abierto (lo cual, tratándose de la interpretación, habría de consistir en la imposibilidad de cerrar o dejar nada clausurado). Lo que nos lleva a que si aplicásemos la sospecha hermenéutica a la propia hermenéutica (lo cual entraña paradojas insolubles) y en concreto a la heideggeriana, veríamos que también Heidegger pretende dar una interpretación verdadera de Nietzsche, la cual considera válida por situarse en un plano no de filosofía tradicional ni de eruditismo, sino en su pretendido plano ontológico, digamos que propuesto como más profundo que los otros, desde el que Heidegger "comprende" la doctrina del eterno retorno, mientras que los demás no la comprenden. La respuesta a "ipor qué Heidegger comprende lo que otros no comprenden?" consiste en decir que ha indagado con mayor profundidad filosófica, hasta la esencia del pensar nietzscheano, mientras que los demás se habrían quedado en la superficie. La lectura edificante, creativa, encaramada sobre la sistemática, muestra aquí su superioridad manifiesta.

Un ejemplo claro de que Rousseau leyó a Platón de manera edificante procede del contraste entre el Discurso sobre las ciencias y las artes y la República,

7 HeIdegGer, op. cit., «La unidad de voluntad de poder, eterno retorno y transvaloración», p. 35 .

${ }^{8}$ Ibidem.

${ }^{9}$ Ibídem. 
ante el cual resulta obvio que el primer autor en el primer texto se dedicó ampliamente a comentar y parafrasear al segundo autor respecto al segundo texto mencionado. ¿Es eso plagio o inspiración? Veamos un ejemplo. Platón nos dice:

A menos que los filósofos reinen en los Estados, o los que ahora son llamados reyes y gobernantes filosofen de modo genuino y adecuado, y que coincidan en una misma persona el poder político y la filosofía, y que se prohíba rigurosamente que marchen separadamente por cada uno de estos dos caminos las múltiples naturalezas que actualmente hacen así, no habrá, querido Glaucón, fin de los males para los Estados ni tampoco, creo, para el género humano; tampoco antes de eso se producirá, en la medida de lo posible, ni verá la luz del sol, la organización política que ahora acabamos de describir verbalmente ${ }^{10}$.

Y Rousseau parafrasea con indudable talento, exponiendo y actualizando lo tomado del ateniense:

Pero mientras el poder esté sólo a un lado, y las luces y la sabiduría solas a otro, raramente pensarán los sabios grandes cosas, más raramente aún las harán bellas los príncipes, y los pueblos continuarán siendo viles, corrompidos y desgraciados ${ }^{11}$.

¿No puede acaso decirse que Rousseau está diciendo "lo mismo" que Platón pero con su hermoso estilo y a través de sus bellas palabras? Desde luego se cuida mucho Rousseau de no limitarse a hacer una cita del pasaje platónico resenado, siendo muy superior esa asimilación y volcamiento que cualquier mención directa y entrecomillada de la fuente en cuestión. Rousseau ha asimilado y digerido al pensador griego, utiliza a Platón para elaborar su propio pensamiento y por eso no necesita mencionarlo, no lo cita.

${ }^{10}$ Platón, Repuiblica V, 473d-e.

11 J. J. ROUSSEAU, Discurso sobre las ciencias y las artes, $2{ }^{2}$ parte, págs. 175-176, en Del contrato social. Sobre las ciencias y las artes. Sobre el origen y los fundamentos de la desigualdad entre los hombres, Alianza Editorial, Madrid, 1988. 
Respecto a las lecturas de Nietzsche realizadas no ya por esos eruditos académicos, que pretenden exponer a los legos el verdadero pensamiento de Nietzsche ahorrándoles la confrontación directa de cada cual con el pensador, sino entre las realizadas por otros pensadores, vemos que Foucault y Deleuze tienen una lectura distinta, quizás en parte antagónica o quizás en parte complementaria, de la de Heidegger. Heidegger habría dado unas lecciones sobre Nietzsche en los años 30 en las que la voluntad de poder otorgaría el fundamento infundamentado que necesitaba el nacionalsocialismo, pero tras darse cuenta de su error, habría publicado sus lecciones ya en 1960 corrigiendo la primacía de la voluntad de poder con una primacía del eterno retorno. Pero Foucault y Deleuze no parecerán detectar que la fuerza productiva del pensamiento de Nietzsche estuviese plenamente en el eterno retorno y su debilidad en la voluntad de poder, como hizo Heidegger en definitiva (aunque Deleuze recoge también, a su manera, como central la idea de eterno retorno), sino que más bien desarrollan el pensamiento de Nietzsche en la dirección de la fundamentalidad de la voluntad de poder, pero distinguiendo entre la voluntad de poder como dominio y la voluntad de poder como potencia (al modo spinozista). Mientras el primer Foucault parece arribar a una atmósfera irrespirable con su reduccionismo de todo a relaciones de dominio y el segundo Foucault parece retirarse a un egoísta cuidado de sí, Deleuze avanzará distinguiendo y desarrollando, a partir del pensamiento de la voluntad de poder de Nietzsche, entre fuerzas reactivas (dominio) y fuerzas afirmativas (potencia), así como entre dos modos de nihilismo, el reactivo y el afirmativo, haciendo hincapié en el vitalismo y la creatividad.

Interesa dejar claro que los pensadores han leído a Nietzsche de maneras diferentes sin llegar a un acuerdo pleno, aunque sí parcial (en cada momento histórico), respecto a lo que en el pensamiento nietzscheano podemos considerar como fuerza productiva, repensable y replanteable a los efectos de su desarrollo y continuación, y lo que podemos considerar como debilidad, esto es, lo que hay que rechazar. Los modos de leer a Nietzsche sólo serán inconmensurables cuando sean totalmente contradictorios, no cuando se puedan agrupar como partes de un todo, ni cuando puedan considerarse complementarios, pero para eso hay que poder hablar de comprensión verdadera y de falsa comprensión, lo que resulta muy difícil desde la consideración de la verdad como apertura. Ya Heidegger nos enseña con su lectura que lo fundamental es leer a Nietzsche, siendo secundario el leer a los eruditos e incluso, podríamos añadir, la propia lectura de Heidegger: 
Renunciamos aquí a señalar y, más aún, a comentar las muy variadas obras que se han escrito sobre Nietzsche, ya que nada de ello podría servir al propósito de estas lecciones. Quien no reúna el valor y la perseverancia de pensamiento necesarios para aventurarse en los propios escritos de Nietzsche, tampoco necesita leer nada sobre él ${ }^{12}$.

El propósito de las lecciones de Heidegger, como el de cualquier pensamiento sobre un pensamiento, es el de rescatar y proseguir lo fructífero y detectar, dejándolas de lado, las debilidades. Pero es discutible qué es lo fructífero y qué es lo débil. Desde el paradigma hermenéutico todo puede ser fructífero o débil, y lo fructífero en una época puede ser débil en otra, es más, los eruditos, quienes estudian filológicamente a Nietzsche intentando rescatar la verdad de su pensamiento a través del establecimiento de la verdad histórica, tampoco se ponen de acuerdo y hay no pocas divergencias entre sus lecturas. La sombra de un Nietzsche y un Heidegger como fuentes ideológicas del fascismo planea sobre nuestras cabezas, pese a que el pensamiento francés desde los años 60 del pasado siglo rescatase a ambos pensadores para la reflexión de causas más nobles que las de los nazis.

En el fondo no hay mucha diferencia entre la lectura hermenéutica y la lectura sistemática, pues ambas insisten en que lo fundamental es ir a las cosas mismas, la lectura directa del texto, siendo las lecturas sobre el texto secundarias. Pero la sistemática tiene por objetivo el desentrañamiento de la verdad del texto, mientras que la edificante, queriendo realizar una lectura que no presupone una verdad del texto, lo que logra es tomarse mayores licencias a la hora de utilizar de forma edificante los textos de otros. Al señalar tanto a la edificación como al sistema como presupuestos de lectura de los textos filosóficos partimos de y arribamos a su inclusión bajo el paradigma hermenéutico, que es la koiné filosófica contemporánea, sin por ello asegurar que no pueda retornar y no siga viva, al mismo tiempo, la lectura sistemática, que tendría quizás en Husserl a su último exponente. Sólo si desapareciese el mundo de la fábula desaparecería también el mundo verdadero.

Pero difícilmente desaparecerá la lectura sistemática, al menos mientras haya textos sistemáticos que la sustenten, alienten y alimenten. Los textos más difi-

${ }^{12}$ HeIdegGer, op. cit.. p. 25, "El libro La voluntad de poden”. 
ciles y más profundos de la historia de la filosofía universal no pueden ser leídos, sino que han de ser estudiados con profundidad y comentados rigurosamente, directa y minuciosamente. No se lee el Parménides de Platón, ni la Fenomenología del Espiritu, ni la Ética de Spinoza, esos textos no pueden leerse como se lee una novela, han de estudiarse, luchando por su comprensión línea por línea, volviendo atrás e incluso ayudándose con comentaristas de apoyo. Desde luego no partimos de cero, sino que ha de partirse de la familiaridad con numerosos textos de filosofía y con el hábito de su estudio y análisis. No puede abordar semejante tarea el simple letrado, sino que la lectura de la filosofía pura y dura es una labor para alguien previamente cultivado o habituado. La lectura filosófica presupone algo más que la lectura literaria, y leer filosofía con seriedad requiere lo siguiente (y si no es así creemos que quizás no merece la pena hacerlo):

1. ${ }^{\circ}$ Ir a las cosas mismas (fenomenología), al pensamiento mismo, al tema mismo, pensar, yendo al texto mismo (sea libro o realidad), pero esforzándose línea por línea, forcejeando, y, desde luego, yendo también al original (alemán, griego, latín o a cualquiera que sea la lengua); pues aun si se tienen pocos conocimientos en la lengua en cuestión resulta indispensable e insalvable y siempre provechoso el realizar ese acercamiento. Y para suplir en su caso las deficiencias y mejorar en el conocimiento de la lengua original, no está de menos el ayudarse mediante las traducciones a otras lenguas que se conozcan mejor. Hasta aquí lo esencial de la lectura sistemática de un texto filosófico. Con ello bastaría para la edificación, esto es, para familiarizarse con la filosofía y ser capaz de producir filosofía, con lo cual vemos que lo sistemático puede armonizarse en cierto modo con lo edificante. A menudo lo edificante presupone lo sistemático, aunque nunca lo garantiza. Excepcionalmente podrán omitirse algunos pasos y, por así decirlo, dar un salto, entrar directamente en la puerta de los creadores, sin cumplir con los a menudo castradores rituales de erudición que parece exigir el culto al genio.

2. Ir hacia quienes hayan realizado un trabajo semejante, tener trato y conversación con ellos.

3. Habría ya que situar los comentarios al texto dentro de la producción fundamental del autor en cuestión, e incluso hacer dialogar a la obra concreta y autor en relación con los demás grandes pensadores, señalando sus afinidades, discrepancias y localizando cuándo otro pensador puede 
esclarecer el estudiado, apoyándose para ello en toda la tradición de las fuentes primarias. Por eso Gadamer insistirá en que la tarea hermenéutica es infinita y que un texto es ese mismo texto más todas sus interpretaciones. Con estos dos o tres primeros puntos bastaría para pensar y se cumpliría con el trabajo propiamente filosófico, que es aquel que nos puede formar y permitir llegar a pensar por nosotros mismos.

Pero aún resta un cuarto y último punto, que no por ser el menos elevado será el menos importante, pues de él dependerá la educación y, con ella, también en alguna medida la emergencia de los otros puntos señalados. Un punto importante a la hora de la lectura de los textos filosóficos, pero que, a su vez, supone un cuarto elemento dentro de los peligros que impiden el tránsito desde el comentarista de los textos ajenos al pensador propiamente dicho.

4. Mesianismo, vanidad y temor son los tres elementos más entorpecedores del camino del pensar. El primero indica que se toma un texto como si fuese la Biblia, realizándose una especie de exégesis confesional cuando se produce una empatía total con el pensador estudiado y se defiende su obra como si fuese literatura revelada. No en vano la hermenéutica se inauguró cuando pensadores-filólogos como Schleiermacher, el hacedor de velos, se dedicaron a la labor filológica, contraria de lo que mienta su nombre, de desmitologizar la Biblia, desvelando su contextualidad y destruyendo con ello su hechizo. La vanidad indica el fenómeno de la identificación con la figura mesiánica y el temor explica el enamoramiento y la hipnosis que produce en el creyente el texto revelado. Entonces, vemos que en la filosofía misma puede residir un peligro, un riesgo que sólo podrá ser conjurado mediante su contextualización (pues no sólo en la veneración erudita existirá el peligro de la glosa eterna, sino que en el pensar mismo existirá el peligro de tornarse esclavo de un solo pensar).

$Y$ es que si se quiere completar el rigor filosófico con rigor filológico y tomar el segundo como educación propedéutica para el primero, habría que hacerse cargo de la bibliografía secundaria existente, señalando tradiciones de interpretación entre los comentaristas más reputados e intentando seleccionar lo más relevante dentro de la historiografía para inscribir el texto en el contexto histórico en el que fue realizado. Apoyándonos en la bibliografía secundaria existen- 
te habríamos de determinar lo relevante y detectar e inscribir el texto en el contexto histórico en el que fue realizado, separando luego lo meramente contextual de lo propiamente filosófico y atendiendo igualmente al marco o contexto histórico desde el cual nosotros mismos nos enfrentamos al texto y en el que estamos inmersos. Pero al no leer los textos filosóficos como los leen los filósofos, al no atrevernos a pensar en los temas que planean sobre los textos y contentarnos con la colección de datos, habríamos de sustituir lo que sería nuestro propio acto de pensar con el rigor filológico y abundar de por vida en este punto cuarto, el dedicado a la literatura secundaria, que en el peor de los casos se reduciría a la conversión en lector de reseñas, sin que se llegase a realizar ese tránsito por los cuatro tiempos en el espacio de lectura de los textos filosóficos que nos llevase a pensar por nuestra cuenta. Eso es lo que ocurre cuando la Academia platónica se vuelve helenística y cuando llega a padecer el mal endémico de la enfermedad histórica, el peligro de terminar atendiendo exclusivamente a este cuarto punto - por motivos crematísticos, pues es en definitiva lo que la sociedad en que vivimos demanda y remunera - creyéndose entonces, ilusoriamente, que se pueden ahorrar los tres primeros. Con lo cual el pensar acaba, en tal caso, desapareciendo de la labor pedagógica y del horizonte de la producción social, adviniendo con ello un tiempo de nihilismo, de ausencia de pensamiento. Y es que se puede decir que lo ideológico ha vencido cuando todos los caminos del pensar desembocan en el nihilismo, conciencia vacía de un mundo regido por el valor de cambio, ante la cual ya no sabríamos preguntar al texto ni desvelar la pregunta originaria que le dio origen.

Todo ello, el conjunto de esa labor de lectura hermenéutica en cuatro fases, que si queda truncada y parcial a veces nos impide llegar a pensar, tanto como si se completa, ¿para qué sirve?, ¿¿cuál es la finalidad de quien la aborda? ¿No será la de capacitarse para la realización de algo semejante a lo que los grandes o pequeños filósofos realizaron? ¿No estaremos, en el mundo de la lectura de los textos filosóficos, tomando los medios por los fines?

Hay muchas clases de filósofos y de filosofías, los hay mundanos y los hay académicos, los hay sistemáticos y los hay edificantes, los hay grandes y los hay pequeños. No hay que exagerar en esto ni ser excesivamente purista, ya que la labor hermenéutica más modesta y la reflexión meramente incipiente, valga la presente, ya es muestra de trabajo y creatividad, entrañando pensamiento y siendo por ello loable. 
Pero si queremos evitar los peligros que acechan a quien aborda la lectura de los textos filosóficos no hemos de perder de vista que el objetivo es el pensar y que lo más importante es el pensamiento, aunque la filosofía acabe reducida a un ámbito extremadamente minoritario, quizás ya desaparecido o en vías de extinción. 